\title{
Damage Mechanisms in Silicon-Molybdenum Cast Irons Subjected to Thermo-mechanical Fatigue
}

Viktor Norman, Peter Skoglund, Daniel Leidermark and J ohan Moverare

The self-archived version of this journal article is available at Linköping University Electronic Press:

http:// urn.kb.se/ resolve?urn=urn:nbn:se:liu:diva-137287

N.B.: When citing this work, cite the original publication.

Norman, V., Skoglund, P., Leidermark, D., Moverare, J., (2017), Damage Mechanisms in Silicon-

Molybdenum Cast Irons Subjected to Thermo-mechanical Fatigue, International J ournal of Fatigue, 99(2), 258-265. https:// dx.doi.org/ 10.1016/j.ijfatigue.2017.01.014

Original publication available at:

https:// dx.doi.org/10.1016/j.jjfatigue.2017.01.014

Copyright: Elsevier

http:// www.elsevier.com/

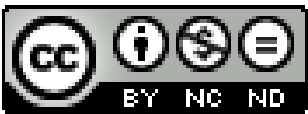




\title{
Damage Mechanisms in Silicon-Molybdenum Cast Irons subjected to Thermo-Mechanical Fatigue
}

\author{
V. Norman ${ }^{\mathrm{a}, *}$, P. Skoglund ${ }^{\mathrm{a}, \mathrm{b}}$, D. Leidermark ${ }^{\mathrm{c}}$, J. Moverare $^{\mathrm{a}}$ \\ ${ }^{a}$ Division of Engineering Materials, Department of Management and Engineering, \\ Linköping University, SE-58183 Linköping, Sweden \\ ${ }^{b}$ Scania $C V$ AB, Materials Technology, SE-15187 Södertälje, Sweden \\ ${ }^{c}$ Division of Solid Mechanics, Department of Management and Engineering, Linköping \\ University, SE-58183 Linköping, Sweden
}

\begin{abstract}
The damage mechanisms active in silicon-molybdenum cast irons, namely EN-GJS-SiMo5-1 and SiMo1000, under thermo-mechanical fatigue and combined thermo-mechanical and high-cycle fatigue conditions have been investigated. The studied load conditions are those experienced at critical locations in exhaust manifolds of heavy-vehicle diesel engines, namely a temperature cycle of $300-750{ }^{\circ} \mathrm{C}$ with varied total mechanical and high-cycle fatigue strain ranges. It is established that oxide intrusions are formed in the early life from which macroscopic fatigue cracks are initiated close to the end-of-life. However, when high-cycle fatigue loading is superimposed, small cracks are preferentially initiated at graphite nodules within the bulk. In addition, it is found that both the oxidation growth rate and casting defects located near the surface affect the intrusion growth.
\end{abstract}

Keywords: Cast iron, Thermo-mechanical fatigue, High-cycle fatigue, Environmental assisted fatigue, Fatigue crack growth

\section{Introduction}

The heavy-vehicle automotive industry is constantly subjected to higher demands regarding exhaust emission and power efficiency; the main reason being the progressive restraining in carbon and toxic emission through new

\footnotetext{
*Corresponding author. Phone: 004613284695

Email address: viktor.norman@liu.se (V. Norman) 
European Union directives. To meet these requirements, heavy-vehicle engine manufacturers are enforced to achieve a higher combustion temperature and pressure, which increases the thermal and mechanical loads on the engine parts enclosing the combustion chamber and the exhaust gas flow. As a consequence, the engine parts become more susceptible to fatigue failure associated with the start-operate-stop cycle, i.e. the recurrent heating and cooling of the engine parts due to the shut down and restart in every-day engine operation [1]. Thus, there is a great industrial interest to find answers to how manufacturers can further push the efficiency without the risk of reduced engine durability.

The above issues have led to an increased activity in evaluating the fatigue resistance of both already employed and potential materials [2, 3], which in the case of heavy-vehicle diesel engines most commonly consist of different cast irons grades, cast steels and occasionally cast aluminium. At present, the mentioned evaluation is performed through simplified laboratory tests constructed to capture the main characteristics of the real loading case scenario. The tests are uniaxial fatigue tests, often referred to as thermo-mechanical fatigue (TMF) and combined thermo-mechanical and high-cycle fatigue (TMF-HCF) tests due to the involvement of a variational temperature in contrast to conventional low-cycle fatigue (LCF) tests [2-4]. The latter of the two, which is a more complicated version of a TMF test where the mechanical loading is superimposed with a high frequent strain load, is employed in order to see the effect of adding vibrations. The outcome of these results is not only to compare materials, but also to guide the design of engine components to be more robust [1, 5]. However, the material properties which control the TMF behaviour are often unclear or unstudied, hence there is a need to study the microstructural aspects and their influence on the TMF resistance in order to know in what way materials should be developed. In other words, knowledge about how material properties and the TMF behaviour are related will reveal how engine material should be selected and processed in order to maximise the performance and durability.

The attention of this study is concentrated on the exhaust manifold, which is a component known to be exposed to severe thermo-mechanical loading, and an associated material often used in their manufacturing process, namely the silicon-molybdenum spheroidal graphite iron EN-GJSSiMo5-1 [6-8]. In addition, a newly developed silicon-molybdenum cast iron, SiMo10000, is included in the investigation due to its high potential as 
an exhaust manifold material [9]. The central purpose of the present investigation is to identify the underlying fatigue damage accumulation mechanism occurring in these materials, as well as the microstructural properties affecting it, at critical load conditions present in these components. In this way, clues about how these materials can be modified in order to constrain the damage accumulation rate are uncovered. To this end, the TMF and TMF$\mathrm{HCF}$ resistance at relevant temperatures and mechanical load conditions have been characterised and related to the microstructure of the investigated materials. As a consequence, it is now clear which microstructural properties are important for the TMF resistance, which in turn can be applied in the manufacturing and processing to further improve the durability of exhaust manifolds.

\section{Materials and experimental procedure}

\subsection{Materials and specimens}

The investigated materials were SiMo1000 and two grades of EN-GJSSiMo5-1, henceforth abbreviated as SiMo51, which are materials commonly associated with exhaust manifolds [6-9]. The former is a recently developed silicon-molybdenum cast iron with aluminium, marketed by George Fischer Eisenguss GmbH [9], while the latter two are standard materials often employed in the heavy-vehicle automotive industry. All materials are ferritic and nodular, with some exceptions for SiMo1000 which contains a significant fraction of the compacted graphite shape and a high amount of iron and aluminium rich carbides [9]. Regarding the two SiMo51 grades, they were cast with different cooling rates by employing two different mould thicknesses, $16 \mathrm{~mm}$ and $20 \mathrm{~mm}$, giving $3.5^{\circ} \mathrm{C} / \mathrm{s}$ and $2.5^{\circ} \mathrm{C} / \mathrm{s}$ cooling rate respectively. In order to separate the two grades, SiMo51 SC denotes the SiMo51 obtained with the slow cooling rate and SiMo51 RC with the rapid cooling rate. The SiMo51 materials are also heat-treated, namely a solution treatment at $900^{\circ} \mathrm{C}$ and a subsequent normalisation, in order to remove potential pearlite content. The chemical composition of each material is displayed in Table 1 and the typical appearance of the microstructure is shown in Fig. 1. These images were taken from regions cut out from the parallel section of untested TMF specimens.

The static mechanical properties are displayed in Table 2 obtained from tensile tests at room temperature. The tensile tests were conducted according to the ISO 6892-1:2009 A222 standard in an Instron 5582 electromechanic tensile test machine equipped with a $100 \mathrm{kN}$ load cell. The strain 
was monitored with an Instron extensometer (cat.no. 2620-601) with a 12.5 $\mathrm{mm}$ gauge length. The specimens were cylindrical with $7 \mathrm{~mm}$ diameter, 28 $\mathrm{mm}$ parallel length, $7 \mathrm{~mm}$ transition radius and a total specimen length of $116 \mathrm{~mm}$.

Table 1: Chemical composition in weight percent of SiMo51 SC, SiMo51 RC and SiMo1000. The iron content is implicit.

\begin{tabular}{lccccccccccc}
\hline & $C$ & $S i$ & $M n$ & $S$ & $P$ & $N i$ & $M o$ & $C u$ & $S n$ & $T i$ & $A l$ \\
\hline SiMo51 SC & 3.16 & 4.33 & 0.407 & 0.008 & 0.014 & $<0.050$ & 0.91 & 0.073 & $<0.010$ & 0.017 & 0.017 \\
SiMo51 RC & 3.17 & 4.12 & 0.35 & 0.007 & 0.021 & $<0.050$ & 0.85 & 0.10 & $<0.010$ & 0.016 & 0.020 \\
SiMo1000 & 3.57 & 2.72 & 0.25 & 0.004 & 0.031 & 0.84 & 0.81 & 0.11 & 0.005 & 0.019 & 3.08 \\
\hline
\end{tabular}

Table 2: Mechanical properties of SiMo51 SC, SiMo51 RC and SiMo1000 acquired from tensile tests performed at room temperature.

\begin{tabular}{lcccc}
\hline & $R_{p 0.2}$ & $R_{m}$ & $E$ & $A$ \\
& {$[M P a]$} & {$[M P a]$} & {$[G P a]$} & {$[\%]$} \\
\hline SiMo51 SC & $494 \pm<1$ & $587 \pm 11$ & $164 \pm<1$ & $5.3 \pm 1.2$ \\
SiMo51 RC & $480 \pm 6$ & $592 \pm 4$ & $164 \pm<1$ & $10.5 \pm 1.6$ \\
SiMo1000 & $536 \pm 13$ & $566 \pm 10$ & $157 \pm 2$ & $1.0 \pm 0.4$ \\
\hline
\end{tabular}
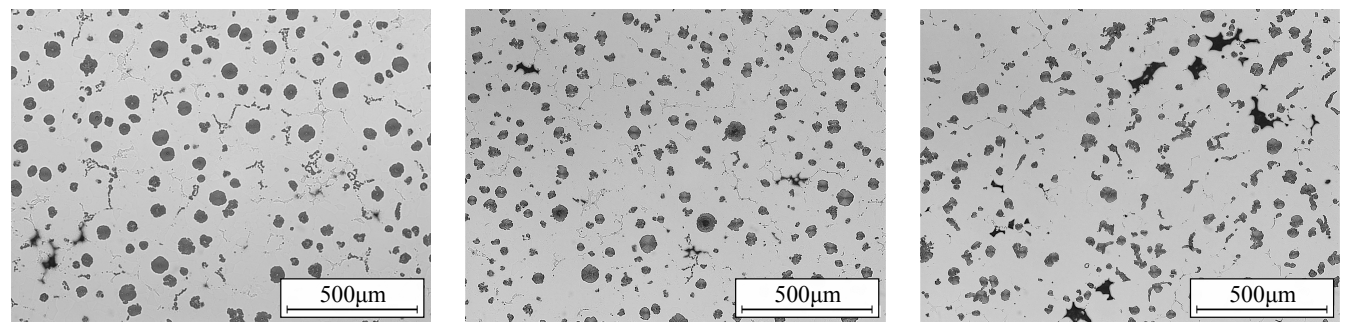

Figure 1: Optical microscope images of the microstructure of (a) SiMo51 SC, (b) SiMo51 $\mathrm{RC}$ and (c) SiMo1000.

\subsection{Thermo-mechanical fatigue tests}

A thermo-mechanical fatigue test is a generalised LCF test in which the temperature is periodically varied together with the controlled mechanical load variable [4], which most commonly is represented by an applied mechanical strain. Naturally, the period of the thermal and mechanical cycle are the same, however different phase shift angles are often considered, such as in-phase and out-of-phase, corresponding to $0^{\circ}$ and $180^{\circ}$ phase shift. 
In a combined TMF-HCF test, a HCF strain component is added on top of the mechanical strain variable defined in a regular TMF test. The uniaxial extensometer strain $\varepsilon_{e}$ can then be written as

$$
\varepsilon_{e}(t)=\varepsilon_{T h}(t)+\varepsilon_{T M F}(t)+\varepsilon_{H C F}(t)=\varepsilon_{T h}(t)+\varepsilon_{M e c h}(t)
$$

where $\varepsilon_{T h}$ is the thermal strain representing the thermal expansion and $\varepsilon_{T M F}$ is the mechanical strain denoted here as the TMF strain which is the base strain component with the same period as the temperature cycle. The $\varepsilon_{H C F}$ variable is the $H C F$ strain corresponding to the high-frequent strain component superimposed on the TMF strain. The combined mechanical strain variable, i.e. the TMF and HCF strain added together, is denoted as the total mechanical strain, $\varepsilon_{\text {Mech }}$, to emphasise that it is composed of two mechanical strain components. The strain variables as well as their respective strain range values are displayed in Fig. 2. For consistency, the mechanical strain range will be referred to as the total mechanical strain range under both TMF and TMF-HCF conditions, even though it only consists of the TMF strain range in the former case.

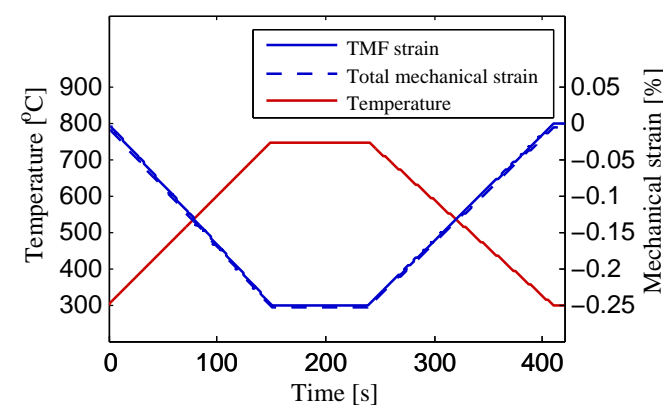

(a)

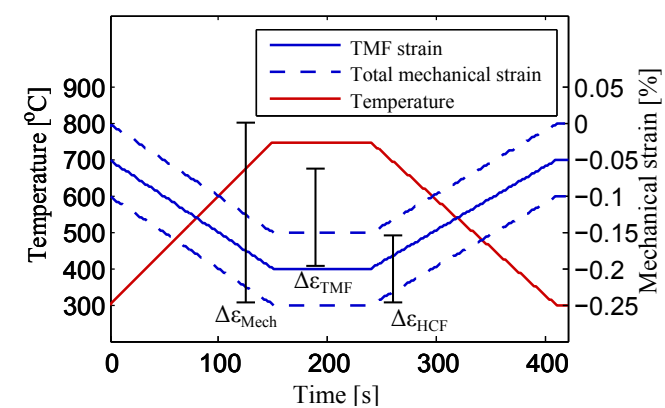

(b)

Figure 2: Examples of the employed mechanical strain and temperature cycle in the (a) TMF and (b) TMF-HCF tests. The total mechanical strain is represented by the envelope generated by the HCF oscillation about the TMF strain. The HCF, TMF and total mechanical strain range are also marked out in (b).

All TMF and TMF-HCF tests performed in this study were conducted in an out-of-phase configuration with the temperature going between 300 and $750{ }^{\circ} \mathrm{C}$. Furthermore, the maximum total mechanical strain was fixed to $0 \%$ and the HCF strain was always chosen to oscillate symmetrically about the TMF strain, see Fig. 2. Accordingly, the studied variables were 
the total mechanical strain range and the HCF strain range, leaving the TMF strain as a free variable. The reason for this is the desire to study the isolated life-reducing effect of an applied HCF load without the influence of the increase in the total mechanical strain range, and the resulting increase in the maximum stress, which otherwise would be inferred if the TMF strain was to be kept constant. For an extended discussion on this, see Norman et al. [10]. The TMF cycle consisted of $150 \mathrm{~s}$ heating up and $170 \mathrm{~s}$ cooling down as well as hold times at the maximum and minimum temperature, namely $90 s$ and $10 s$ respectively. Moreover, the HCF frequency was chosen as $15 \mathrm{~Hz}$, motivated by the rotational speed of the crank shaft. The tested temperature cycle and the total mechanical strain load were selected to be similar to the conditions found in exhaust manifolds in field use or in accelerated testing.

The TMF and TMF-HCF tests were carried out in an Instron 8801 servo hydraulic test machine, in which the specimens were heated by induction heating using an encircling copper coil. The cooling of the specimen was accomplished by natural convection assisted by a compressed air flow directed towards the parallel section through three nozzles. The tests were controlled using a dedicated TMF software developed by Instron and the extensometer strain was measured using an Instron extensometer with a $12.5 \mathrm{~mm}$ gauge length.

Smooth cylindrical test specimens were used whose geometry was defined by a $6 \mathrm{~mm}$ diameter, $25 \mathrm{~mm}$ parallel length, $30 \mathrm{~mm}$ transition radius and a total specimen length of $145 \mathrm{~mm}$. The specimens were gripped using hydraulic grips.

Following the validated code-of-practice [4], a failure criterion to define the number of cycles to failure $N_{f}$ was chosen as the $2 \%$ stress deviation from the tangent drawn at the last point of zero curvature in the stress range curve.

\subsection{Metallographic investigation}

Metallographic investigations were conducted on both untested as well as interrupted TMF and TMF-HCF tests. All cross-sectional samples were taken from the parallel section of TMF specimens and investigated in a Nikon Optiphot optical microscope, using magnifications ranging from 100 to 1000 . Regarding the interrupted tests, the specimens were cut along the longitudinal axis, and then cut again in the cutting plane perpendicular to the first, in order to acquire one more cross-sectional surface. The metallographic samples were polished using a standard program for cast irons. 
Microstructural features were characterised by investigating cross-sections, namely the microshrinkage pores size in untested materials and the length of oxide intrusions in interrupted TMF and TMF-HCF specimens. The pore size was determined as the diameter of the smallest possible circle able to contain the defect. In the interrupted specimens, the oxide intrusion length was assessed by measuring the average depth of oxidation penetration following the ISO 26146:2012(E) standard. More precisely, the oxidation depth was measured from the outer edge of the oxide scale to the innermost interface between the matrix and the scale, at regular intervals axially along the specimen surface within the gauge length. The parameters were measured for all materials and over two different cross-sections of each material. The average and standard deviation of the microshrinkage pore sizes reported here only includes pores with a diameter larger than $50 \mu \mathrm{m}$; a length typically in the order of the diameter of graphite nodules. Similarly, the density of microshrinkage pores was reported as the total number of pores with a diameter larger than $50 \mu \mathrm{m}$ divided by the studied area. Regarding the oxidation penetration depth, all measurements were included in the average and standard deviation.

\section{Results and discussion}

\subsection{TMF and TMF-HCF characterisation}

Previous studies have demonstrated that the fatigue life of many different cast irons, namely lamellar (LGI), compacted (CGI) and spheriodal graphite iron (SGI), can be associated with small fatigue crack propagation and coalescence, both under LCF and TMF conditions for temperatures below $500^{\circ} \mathrm{C}[3,10-16]$. In the case of SGI, small cracks are most frequently initiated at microshrinkage pores under these circumstances [3, 14, 17], and possibly also at graphite nodules [11, 15].

For the above reason, the microstructure of as-received specimens were investigated before conducting the TMF experiments, in particular regarding the microshrinkage pore size and distribution, since it was hypothesised that these would influence the TMF properties. Examples of such microshrinkage pores in the studied materials can be seen in Fig. 1, while the maximum and average microshrinkage pore size as well as the density are displayed in Table 3. Apparently, the SiMo51 grades are similar in this regard except for slightly larger defects on average in SiMo51 SC, about 20 $\%$ in average size, while SiMo1000 has almost three times more defects. 
Table 3: Maximum and average size of microshrinkage pores as well as the density of pores larger than $50 \mu m$ in diameter, of SiMo51 SC, SiMo51 RC and SiMo1000.

\begin{tabular}{lccc}
\hline & $\begin{array}{c}\text { Maximum size } \\
{[\mu \mathrm{m}]}\end{array}$ & $\begin{array}{c}\text { Average size } \\
{[\mu \mathrm{m}]}\end{array}$ & $\begin{array}{c}\text { Density } \\
{\left[\mathrm{mm}^{-2}\right]}\end{array}$ \\
\hline SiMo51 SC & 278.7 & $92.0 \pm 56.0$ & 0.80 \\
SiMo51 RC & 143.4 & $75.4 \pm 23.8$ & 0.90 \\
SiMo1000 & 282.9 & $96.5 \pm 47.5$ & 2.34 \\
\hline
\end{tabular}

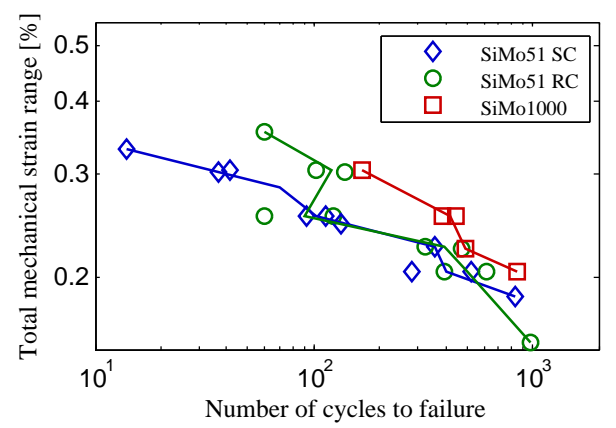

(a)

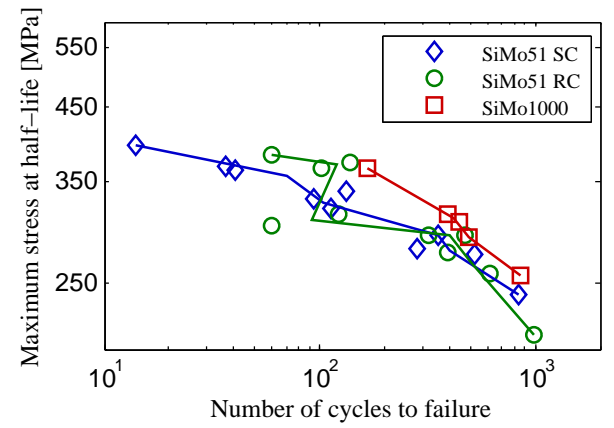

(b)

Figure 3: (a) Strain-life and (b) stress-life curves of TMF tests with $300-750{ }^{\circ} \mathrm{C}$ temperature cycle and without HCF loading, for SiMo51 SC, SiMo51 RC and SiMo1000.

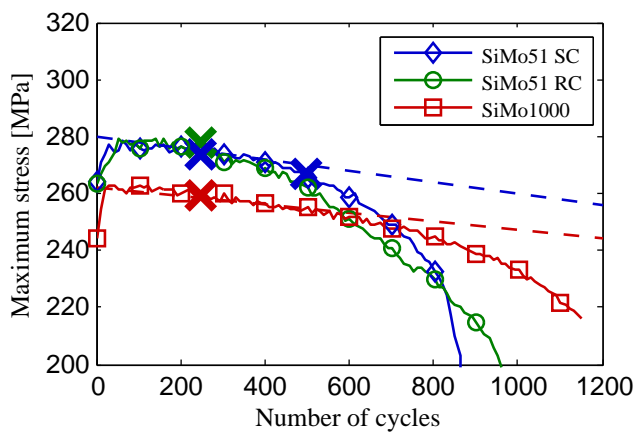

Figure 4: (a) Maximum stress curves measured in a TMF test with $300-750^{\circ} \mathrm{C}$ temperature cycle and $0.20 \%$ total mechanical strain range of SiMo51 SC, SiMo51 RC and SiMo1000. Tangent lines are drawn to illustrate the linear stress-decrease regime of the TMF test and the cycles at which the interrupted TMF tests have been investigated are each marked with a cross.

The acquired strain-life and stress-life curves for the TMF tests with the temperature cycle $300-750{ }^{\circ} \mathrm{C}$ are shown in Fig. 3. The latter plot is ac- 
quired by plotting the number of cycles to failure versus the maximum stress at half-life. Surprisingly, the TMF properties of the three materials were not in accordance with what was first anticipated from the microshrinkage pore characterisation, Table 3. Clearly, SiMo1000 is superior to the SiMo51 grades, even though it has more microshrinkage pores. Furthermore, it is also established that changing the cooling rate of SiMo51 does not have any significant effect regarding TMF properties, except for longer lives at high total mechanical strain ranges. Consequently, it appears as there is no explicit correlation between the TMF resistance and the occurrence of defects, in contrast to what was first anticipated.

The above conversion from the total mechanical strain range to a stress variable by taking the maximum stress at mid-life is motivated by the fact that the maximum stress is fairly constant during a TMF tests; only about 10 to $20 \mathrm{MPa}$ net decrease during the fatigue life as seen in Fig. 4. Comparing Figs. 3a and 3b reveals no greater difference, except for a slightly less scatter in the stress-life curve. Thus, it seems that the maximum stress manifests a better correlation with the number of cycles to failure than the applied total mechanical strain range.

The lower maximum stress in SiMo1000 compared to the SiMo51 grades seen in Fig. 4 is attributed to the better mechanical properties of the former at elevated temperatures. For instance, the reduction in ultimate tensile strength at elevated temperatures occurs for a higher temperature in SiMo1000 compared to SiMo51 [9]. Thus, since SiMo1000 has a better resistance to softening at high temperatures, the stress relaxation at the compressive side of the first TMF cycle is less, see Fig. 5. Consequently, SiMo1000 starts at a lower stress level when going back in tension at the start of the second half-cycle, which results in a lower maximum stress for all subsequent cycles compared to SiMo51 SC and SiMo51 RC.

Fig. 6 presents the maximum stress evolution in TMF-HCF tests of SiMo51 SC for different HCF strain ranges. From this figure, it is clearly indicated that the maximum stress is reduced due to the superimposed $\mathrm{HCF}$ cycling. This is a known effect in TMF-HCF testing that has been discussed in a previously published paper [10], which most likely is due to suppression of dynamic recovery that otherwise would have occurred at the maximum temperature if not for the HCF cycling. As a consequence, a slightly larger compressive stress is attained at the hot side of the TMF cycle, i.e. the lefthand side of the hysteresis loop in Fig. 5, which results in a lower maximum stress at the opposite side. 


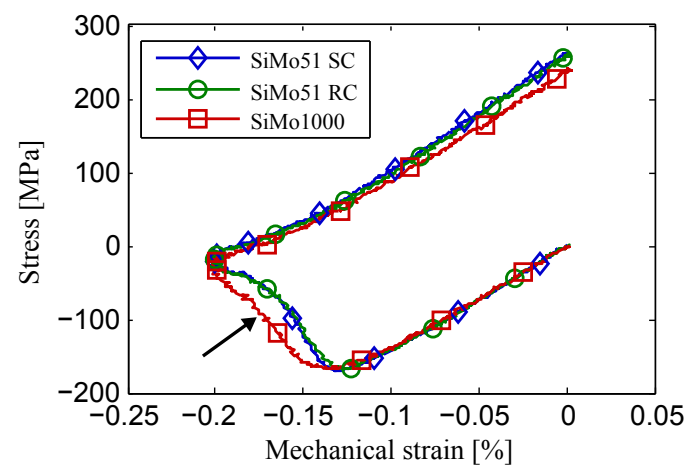

Figure 5: Hysteresis loops of the first cycle of a TMF test with $300-750{ }^{\circ} \mathrm{C}$ temperature cycle and $0.20 \%$ total mechanical strain range of SiMo51 SC, SiMo51 RC and SiMo1000. The arrow points out the mentioned difference in stress relaxation during the first compressive half-cycle, which is suggested to be the reason for a lower maximum stress in SiMo1000 in subsequent cycles compared to SiMo51 SC and SiMo51 RC.

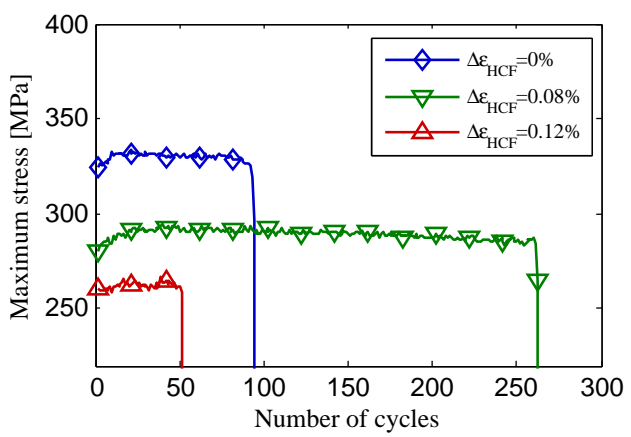

Figure 6: Maximum stress curves of SiMo51 SC measured in a TMF-HCF test with $300-750{ }^{\circ} \mathrm{C}$ temperature cycle, $0.25 \%$ total mechanical strain range and different $\mathrm{HCF}$ strain ranges.

A relevant property in the industrial context is the TMF-HCF threshold $[3,10]$, which relates how resistant a material is to superimposed HCF in a TMF test. This property can be defined for materials possessing a clear threshold value in the HCF strain range above which the fatigue life is significantly reduced. In fact, many different materials, e.g. cast aluminium $[18,19]$, cast irons $[3,10,15,20]$ and superalloys $[2,21]$, have been reported to exhibit such a behaviour. Following the previous investigations of Norman et al. [3, 10], the TMF-HCF threshold is here defined as the HCF strain range at which the life measured in a regular TMF test is reduced to half. It should be noted that this TMF-HCF threshold definition is a 


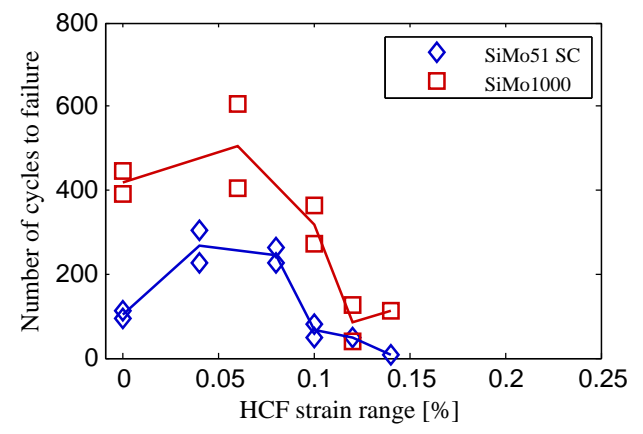

(a)

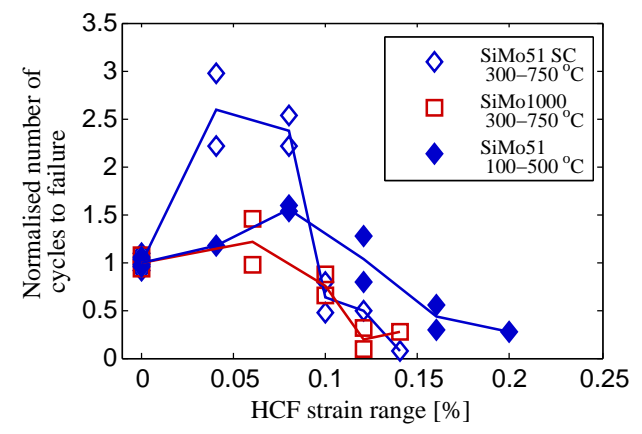

(b)

Figure 7: (a) TMF-HCF plot and (b) normalised TMF-HCF plot including SiMo51 SC and SiMo1000 conducted with $300-750{ }^{\circ} \mathrm{C}$ temperature cycle and $0.25 \%$ total mechanical strain range. TMF-HCF tests of SiMo51 conducted at $100-500{ }^{\circ} \mathrm{C}$ with $0.44 \%$ and $0.58 \%$ total mechanical strain range, previously presented in [3], are added to (b) for comparison.

crude measure, allowing $50 \%$ life reduction, however it is distinct and easily identified even for TMF-HCF test series with few tested HCF strain ranges.

The TMF-HCF threshold is best illustrated in a TMF-HCF plot, Fig. $7 \mathrm{a}$, in which the number of cycle to failure is plotted as a function of the HCF strain range for SiMo51 SC and SiMo1000 with constant total mechanical strain range of $0.25 \%$, as explained in Section 2.2. Normalising the TMF$\mathrm{HCF}$ plot to the fatigue life at zero HCF strain range shows that the TMFHCF threshold of SiMo51 SC and SiMo1000 are almost identical, namely about $0.11 \%$, see Fig. $7 \mathrm{~b}$. On the other hand, it must be remembered that the absolute value of the fatigue life is longer for SiMo1000 even though the TMF-HCF threshold is the same, see Fig. 7a.

Fig. 7 also includes the data obtained for SiMo51 with the temperature cycle $100-500{ }^{\circ} \mathrm{C}$, previously presented in [3]. Apparently, the TMF-HCF threshold is slightly lower for the $300-750{ }^{\circ} \mathrm{C}$ temperature cycle, which could be due to a number of reasons. The most plausible explanation is given by considering the number of HCF cycles per TMF cycle which are applied during tensile stresses. Clearly, the TMF-HCF threshold must be dependent on the HCF frequency, the TMF cycle period and on how the hysteresis loop is located with reference to the zero stress line. The HCF cycles applied during compressive stresses are disregarded since it is presupposed that the cracks are closed, which was demonstrated to be a valid in the case of EN-GJV-400 [10]. Effectively, even though the TMF cycle is shorter in the $300-750{ }^{\circ} \mathrm{C}$ 
tests, $420 s$ compared to $450 s$, the number of HCF cycles applied at tensile stress is higher, about 6000 compared to 4000 in the $100-500{ }^{\circ} \mathrm{C}$ test condition [3]. Thus, since the number of HCF cycles contributing to fatigue crack propagation is higher, it is natural that the TMF-HCF threshold is lowered in the $300-750{ }^{\circ} \mathrm{C}$ case. Other possible influences could be the temperature dependence in the elastic modulus which affects the HCF stress range, as well as the difference in the total mechanical strain range. Nevertheless, the above given explanation regarding the number of HCF cycles is still considered to be the most likely. The above argumentation is reflected in the expression of the TMF-HCF threshold derived from the model presented in [3].

By further inspection of Fig. 7, it is also noted that the fatigue life is increased for intermediate $\mathrm{HCF}$ strain ranges; up to a factor of three in the case of SiMo51 SC. This can be explained by the apparent decrease in maximum stress as the HCF strain range is increased, see Fig. 6. Thus, since the HCF strain range is supposedly below the TMF-HCF threshold and the maximum stress is comparably lower, the fatigue life is expected to be longer for intermediate HCF strain ranges. As a matter of fact, the gain in lifetime is well accounted for by comparing the maximum stress in Fig. 6 with the trend seen in the stress-life curve, Fig. 3b. As seen in the latter figure, a reduction of $50 \mathrm{MPa}$ in maximum stress for SiMo51 SC which is caused by application of $0.08 \% \mathrm{HCF}$ strain range, Fig. 6, roughly corresponds to an increase in fatigue life of a factor of three.

\subsection{Fatigue behaviour in TMF and TMF-HCF at high temperature}

In order to have understanding of the fatigue behaviour, TMF tests on SiMo51 SC were conducted which were interrupted before complete failure occurred. The specimen were then subsequently investigated in metallographic studies in order to visualise and evaluate the fatigue damage. The SiMo51 SC curve in Fig. 4 shows the maximum stress evolution of the chosen TMF test for this investigation, namely a total mechanical strain range of $0.20 \%$ without HCF loading, where the cross marks points out the number of cycles at which the tests were interrupted. One test was interrupted at 250 cycles, corresponding to about half of the fatigue life, while a second test was interrupted at 500 cycles, which was close to the end-of-life as defined by the failure criterion presented in Section 2.2. In fact, the intention of the latter test was to observe the material state at an instant close to when the maximum stress begins to deviate from a linear decline, as seen in Fig. 4. 


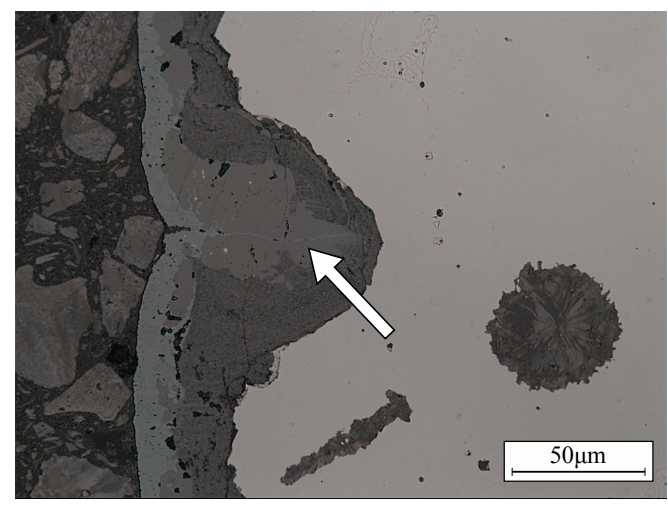

(a)

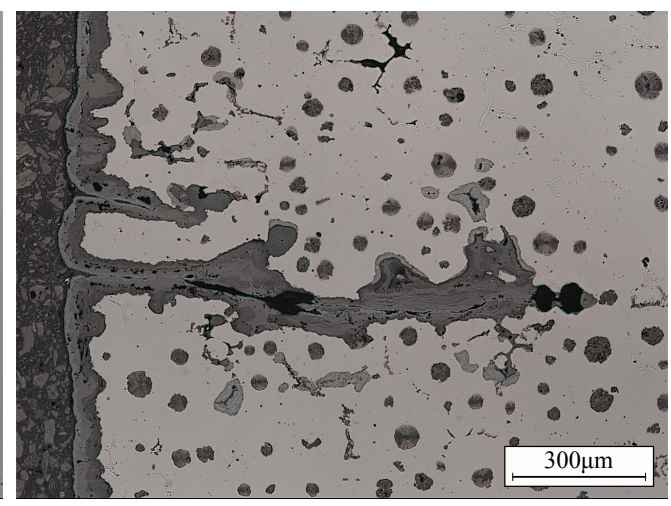

(b)

Figure 8: Oxide intrusion and surface crack observed in SiMo51 SC tested with 300-750 ${ }^{\circ} \mathrm{C}$ temperature cycle, $0.20 \%$ total mechanical strain range and without HCF loading, interrupted at (a) 250 and (b) 500 cycles.

Looking at cross-sections of the interrupted TMF tests suggests that the fatigue behaviour is featured by environmentally-assisted surface cracks. At 250 cycles, oxide intrusions are visible at the surface of the specimen, see the example given in Fig. 8a. At this point, the oxide intrusions are observed in multiple locations at the surface and they are typically about $0.1 \mathrm{~mm}$ long. Most likely, they are initiated through the decarbonisation of graphite nodules located on the surface which results in the replacement of the graphite phase with iron oxides. More importantly, a closer inspection reveals that the oxide intrusions often are fractured, as marked out in Fig. 8 a.

The initiation and growth of oxide intrusions are commonly observed in iron-based materials exposed to TMF conditions [22-24], as well as LCF at elevated temperatures [25-27], and is associated with the recurrent fracture of the oxide scale and subsequent oxidation at the generated crack tip. Following the terminology of Neu and Sehitoglu [22], the surface cracks are categorised as Type I, due to the absence of striation in the oxide scale.

As proposed by many authors [24-26], the oxide intrusions generated during the early cycles are believed to act as fatigue crack initiation sites. This is also motivated in the present study by the investigation of TMF tests interrupted close to the end-of-life at 500 cycles, in which a surface crack has emerged extending over $1.0 \mathrm{~mm}$, see Fig. 8b. Thus, looking at the maximum stress evolution in Fig. 4, the instant at which the maximum stress starts to deviate from a linear regime is interpreted as the same instant 
when a macroscopically large crack is initiated.

As mentioned previously, small crack initiation at casting defects has been shown to be a life-controlling event in EN-GJS-SiMo5-1 in TMF tests conducted with a maximum temperature of $500{ }^{\circ} \mathrm{C}$ [3]. For the 300-750 ${ }^{\circ} \mathrm{C}$ temperature cycle, defect-initiated microcracks are seen to be present as well, however not as dominant as for the $100-500{ }^{\circ} \mathrm{C}$ condition [3] or in comparison to the number and size of the oxide intrusions. Effectively, when going from $100-500{ }^{\circ} \mathrm{C}$ to $300-750{ }^{\circ} \mathrm{C}$, the controlling fatigue mechanism seems to be changed from microcrack growth from internal defects to surface crack growth initiated as oxide intrusions, or possibly a combination of the two. Thus, it is very likely that there is a critical maximum temperature, or temperature range, at which the oxide intrusion growth mechanism comes into play.

As indicated in the previous paragraph, it is possible that microshrinkage pores are still detrimental to the lifetime with the $300-750{ }^{\circ} \mathrm{C}$ temperature cycle. For instance, there have been several examples in this investigation of oxide intrusions passing through microshrinkage pores, filling them with iron oxides. Thus, even though the defects might not induce cracks on their own, they may assist the oxide intrusion to grow deeper into the bulk in less time. Furthermore, it is also possible that the proportion between the significance of the two fatigue crack growth mechanisms is different at different total mechanical strain ranges. This could for instance explain the deviating behaviour of SiMo51 RC when going from $0.25 \%$ to $0.30 \%$ total mechanical strain range in Fig. 3a, where the fatigue life contradictorily appears to increase when increasing the total mechanical strain range. Possibly, this could be a result of a transition of fatigue mechanisms.

Table 4: Maximum and average oxidation penetration depth at 250 cycles in TMF and TMF-HCF tests with $300-750^{\circ} \mathrm{C}$ temperature cycle and $0.20 \%$ total mechanical strain range of SiMo51 SC, SiMo51 RC and SiMo1000. The same mechanical parameters were used in the TMF-HCF test, apart from $0.08 \% \mathrm{HCF}$ strain range.

\begin{tabular}{lcc}
\hline & $\begin{array}{c}\text { Maximum length } \\
{[\mu \mathrm{m}]}\end{array}$ & $\begin{array}{c}\text { Average length } \\
{[\mu \mathrm{m}]}\end{array}$ \\
\hline SiMo51 SC & 216.0 & $98.9 \pm 30.0$ \\
SiMo51 RC & 152.1 & $109.7 \pm 18.3$ \\
SiMo1000 & 194.9 & $90.8 \pm 34.5$ \\
\hline SiMo51 SC, & & \\
TMF-HCF & 232.0 & $96.6 \pm 33.8$ \\
\hline
\end{tabular}




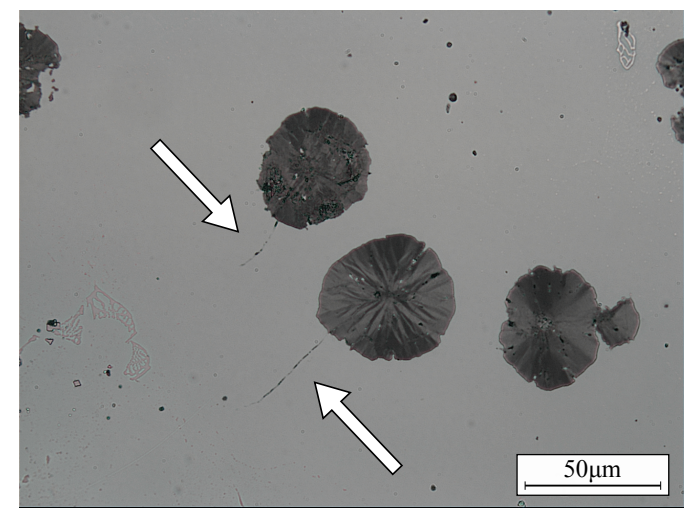

Figure 9: Microcracks observed in SiMo51 SC tested with $300-750{ }^{\circ} \mathrm{C}$ temperature cycle, $0.20 \%$ total mechanical strain range, and $0.08 \% \mathrm{HCF}$ strain range, interrupted at 250 cycles.

The same metallographic experiment was conducted on SiMo51 SC subjected to TMF-HCF with comparable parameters, namely a $300-750{ }^{\circ} \mathrm{C}$ temperature cycle, $0.20 \%$ total mechanical strain range and $0.08 \% \mathrm{HCF}$ strain range, also interrupted at 250 cycles. The comparison of the oxidised surface of the TMF and the TMF-HCF tested specimen does not indicate any significant difference. To support this observation, the typical length of the oxide intrusions were assessed by measuring the average depth of oxidation, as explained in 2.3. The results are given in Table 4, which indicate that the average oxidation penetration depth is almost identical in both conditions. Consequently, the growth of oxide intrusions is proposed to be unaffected by the application of superimposed HCF, at least for this particular HCF strain range.

On the other hand, occurrence of small fatigue cracks emanating from graphite nodules in the bulk are visible in the specimen subjected to TMF$\mathrm{HCF}$, see the example in Fig. 9, in contrast to when subjected to TMF only. By a closer inspection, it was verified that similar cracks were not found in specimens tested under regular TMF conditions. Effectively, it is concluded that the reduction in fatigue life caused by the increase in the HCF loading, see Fig. 7, is due to the increase in microcrack growth occurring within the bulk, rather than accelerated oxide intrusion growth.

\subsection{Material aspects controlling the TMF properties}

Summarising the previous section, it is clear that the fatigue life of SiMo51 SC at the studied load conditions is governed by two fatigue mecha- 
nisms; growth of oxide intrusions at the surface and microcrack propagation from microstructural features. Thus, in order to enhance the performance of silicon-molybdenum cast irons, measures to compensate for these two mechanisms need to be considered.

At 250 cycles in the TMF test with $300-750{ }^{\circ} \mathrm{C}$ temperature cycle and $0.20 \%$ total mechanical strain range, there are signs of oxide intrusions also in SiMo51 RC and SiMo1000. Fig 10 shows examples of intrusions in the two materials which are comparable with the intrusion in SiMo51 SC presented in Fig. 8a. From what can be observed, the fatigue process appears to be very similar in the three studied materials, which also is reflected in the measured oxidation penetration depth, see Table 4 . On average, the oxidation penetration depth is slightly shorter in SiMo1000 while slightly longer in SiMo51 RC compared to SiMo51 SC. However, the difference is small compared to the standard deviation and might therefore not be significant.

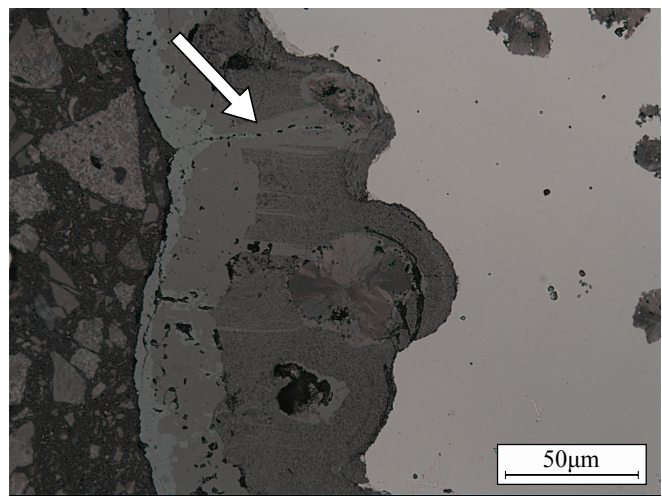

(a)

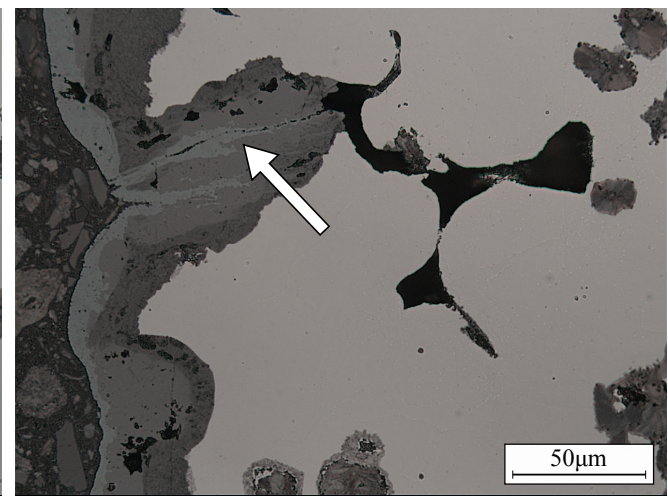

(b)

Figure 10: Oxide intrusions observed in (a) SiMo51 RC and (b) SiMo1000 tested with $300-750{ }^{\circ} \mathrm{C}$ temperature cycle, $0.20 \%$ total mechanical strain and without HCF loading, interrupted at 250 cycles.

It seems likely that the formation of oxide intrusions is governed by two factors, the oxidation resistance and the presence of defects The former is based on the theory proposed by Neu and Sehitoglu [28], which postulates that oxide intrusion growth is driven by the oxidation occurring at the intrusion tip as a consequence of oxide scale rupture. Accordingly, a material which is more resistant to oxidation, i.e. which possess a lower oxide scale growth rate at strain-free conditions, is likely to have a lower oxide intrusion growth rate. Likewise, it is intuitive that a higher density and a larger size of 
microshrinkage pores can aid the formation of longer oxide intrusions when these defects are located at the surface. An example of a defect integrated with an oxide intrusion can be seen in Fig. 10b.

To justify the above argument, an isothermal-exposure oxidation test was carried out to assess the oxidation resistance of the materials, see Fig. 11. Clearly, it is demonstrated that SiMo1000 has the superior oxidation resistance, followed by SiMo51 SC and then SiMo51 RC, which is the same trend indicated in Table 4. The good performance of SiMo1000 is most likely an effect of the high aluminium content, see Table 1, which is known to enhance the oxidation resistance in iron-carbon alloys [29]. The internal difference between the SiMo51 grades is not as easily explained, but could likely also be a result of the differences in the chemical composition.

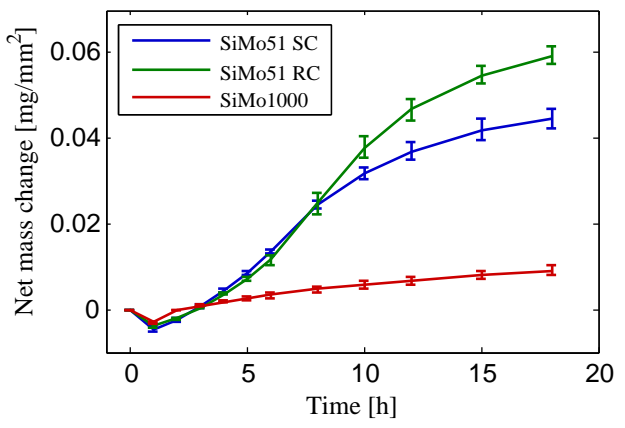

Figure 11: The net mass change versus time measured in an isothermal-exposure oxidation test conducted at $750{ }^{\circ} C$ with SiMo51 SC, SiMo51 RC and SiMo1000. The data points are represented by the average of three samples of each material and the error bars indicate the standard deviation.

However, even though the oxidation resistance of SiMo1000 is significantly superior as seen in Fig. 11, the difference in fatigue life compared to the SiMo51 grades is not as large as expected in view of these isothermalexposure oxidation results. In line with the above proposition regarding the intrusions, this is suggested to be due to the combination of the average defect size and the oxidation resistance, Table 3 and Fig 11 respectively, since defects favour the growth of oxide intrusions while a better oxidation resistance should restrain it. SiMo1000 has a good oxidation resistance but many and large defects, while SiMo51 SC and SiMo51 RC have worse oxidation resistance but on the other hand fewer and smaller defects. Thus, it is possible that the effect of the oxidation resistance is opposed by the presence of defects, which consequently explains why the difference in the 
oxidation penetration depth between the materials is insignificant, see Table 4. Thus, a significant improvement in fatigue life is expected if SiMo1000 could be cast with less defects.

Why the difference in fatigue life between the materials is larger at high maximum stress levels, in contrast to a low values, is however still unclear, see Fig. 3b. As mentioned in 3.2, it is likely that the fatigue mechanisms can be different at different total mechanical strain ranges. For instance, microcrack growth from internal defects might be more important at higher maximum stresses.

\section{Conclusions}

- For a temperature cycle which is experienced at critical locations in the exhaust manifold, namely $300-750{ }^{\circ} \mathrm{C}$, the controlling fatigue crack propagation mechanism in EN-GJS-SiMo5-1 and SiMo1000 consists of environmentally-assisted surface cracks. Initially, small oxide intrusions are formed at the surface which eventually grow into a macroscopically large crack responsible for the final failure.

- The TMF-HCF threshold [3, 10] has been measured to $0.11 \%$ in both EN-GJS-SiMo5-1 and SiMo1000 for the studied TMF conditions, which is close to the value obtained with a $100-500{ }^{\circ} \mathrm{C}$ temperature cycle [3]. In addition, the damage process in TMF-HCF testing consists of small fatigue crack initiation and propagation from graphite nodules rather than accelerated intrusion growth.

- It is indicated that the TMF properties at the studied conditions are affected by the occurrence of microshrinkages and the oxide growth rate, since both factors favour the formation of oxide intrusions. Thus, in order to optimise the durability of silicon-molybdenum cast irons for exhaust manifold applications, these materials should be developed such that the defect size and density are kept at minimum while the oxidation resistance is maximised.

\section{Acknowledgement}

The present study was financed by Scania CV AB, the Swedish Governmental Agency for Innovation Systems (FFI - 2012 - 03625), and the Swedish Foundation for Strategic Research $(S M 12-0014)$. Agora Materiae and the Strategic Faculty Grant AFM (SFO - MAT - LiU\#2009 - 00971) 
at Linköping University are also acknowledged. Special thanks are also addressed to Per Johansson and Peter Karlsson for specimen manufacturing, Patrik Härnman for his technical support on the TMF machine, and the project group at Scania for all their comments and feedback.

\section{References}

[1] S. Trampert, T. Gocmez, S. Pischinger, Thermomechanical Fatigue Life Prediction of Cylinder Heads in Combustion Engines, Journal of Engineering for Gas Turbines and Power 130 (012806) (2008) 1-10.

[2] T. Beck, K. Lang, D. Löhe, Interaction of thermally induced and mechanical fatigue, Transactions of The Indian Institute of Metals 63 (2-3) (2010) 195-202.

[3] V. Norman, P. Skoglund, D. Leidermark, J. Moverare, The effect of superimposed high-cycle on thermo-mechanical fatigue in cast iron, International Journal of Fatigue 88 (2016) 121-131.

[4] P. Hahner, C. Rinaldi, V. Bicego, E. Affeldt, T. Brendel, H. Andersson, T. Beck, H. Klingelhoffer, H. Kuhn, A. Koster, Research and development into a European code-of-practice for strain-controlled thermo-mechanical fatigue testing, International Journal of Fatigue 30 (2) (2008) 372-381.

[5] J. J. Thomas, L. Verger, A. Bignonnet, E. Charkaluk, Thermomechanical design in the automotive industry, Fatigue and Fracture of Engineering Materials and Structures 27 (10) (2004) 887-895.

[6] P. Y. P. Chen, R. Lyons, M. Rakoczy, H. Yamanaka, T. Mimata, Development of the 6.8L V10 heat resisting cast-steel exhaust manifold, in: SAE Technical Paper, 1996.

[7] S. H. Park, J. M. Kim, H. J. Kim, S. J. Ko, H. S. Park, J. D. Lim, Development of a Heat Resistant Cast Iron Alloy for Engine Exhaust Manifolds, SAE Technical Paper (724).

[8] M. Ekström, S. Jonsson, High-temperature mechanical- and fatigue properties of cast alloys intended for use in exhaust manifolds, Materials Science and Engineering A 616 (2014) 78-87.

[9] S. Kleiner, K. Track, SiMo1000 Ein aluminiumlegiertes Gusseisen für Hochtemperaturanwendungen, Giesserei 97 (10) (2010) 28-34.

[10] V. Norman, P. Skoglund, D. Leidermark, J. Moverare, Thermo-mechanical and superimposed high-cycle fatigue interactions in compacted graphite iron, International Journal of Fatigue 80 (2015) 381-390.

[11] P. Clement, J. P. Angeli, A. Pineau, Short Crack Behaviour in Nodular Cast Iron, Fatigue \& Fracture of Engineering Materials and Structures 7 (4) (1984) 251-265.

[12] H. Nisitani, S. Tanaka, Initiation and propagation of fatigue crack in cast iron, Transactions of the Japan Society of Mechanical Engineers, Part A 51 (465) (1985) 1442-1447.

[13] D. Weinacht, D. Socie, Fatigue damage accumulation in grey cast iron, International Journal of Fatigue 9 (2) (1987) 79-86.

[14] Y. Nadot, J. Mendez, N. Ranganathan, Influence of casting defects on the fatigue limit of nodular cast iron, International Journal of Fatigue 26 (2004) 311-319. 
[15] A. Uihlein, K. Lang, D. Löhe, Lifetime Behavior at Superimposed ThermalMechanical and Mechanical Loading, in: Proceedings of the XIth International Congress and Exposition, 2008, pp. 306-313.

[16] M. Endo, K. Yanase, Effects of small defects, matrix structures and loading conditions on the fatigue strength of ductile cast irons, Theoretical and Applied Fracture Mechanics 69 (2014) 34-43.

[17] P. Matteis, G. Scavino, A. Castello, D. Firrao, High-Cycle Fatigue Resistance of Si-Mo Ductile Cast Iron as Affected by Temperature and Strain Rate, Metallurgical and Materials Transactions A 46 (9) (2015) 4086-4094.

[18] T. Beck, D. Löhe, J. Luft, I. Henne, Damage mechanisms of cast Al-Si-Mg alloys under superimposed thermal-mechanical fatigue and high-cycle fatigue loading, Materials Science and Engineering A 468-470 (SPEC. ISS.) (2007) 184-192.

[19] T. Beck, I. Henne, D. Löhe, Lifetime of cast AlSi6Cu4 under superimposed thermalmechanical fatigue and high-cycle fatigue loading, Materials Science and Engineering A 483-484 (1-2 C) (2008) 382-386.

[20] M. Metzger, B. Nieweg, C. Schweizer, T. Seifert, Lifetime prediction of cast iron materials under combined thermomechanical fatigue and high cycle fatigue loading using a mechanism-based model, International Journal of Fatigue 53 (2013) 58-66.

[21] M. Moalla, K. H. Lang, D. Löhe, Effect of superimposed high cycle fatique loadings on the out-of-phase thermal-mechanical fatigue behaviour of CoCr22Ni22W14, Materials Science and Engineering A 319-321 (2001) 647-651.

[22] R. Neu, H. Sehitoglu, Thermomechanical fatigue, oxidation and creep: Part I. Damage mechanisms, Metallurgical Transaction A 20 (9) (1989) 1755-1767.

[23] H. Sehitoglu, Thermo-mechanical fatigue life prediction methods, in: Advances in Fatigue Lifetime Predictive Techniques , ASTM STP 1122, Philadelphia, 1992, pp. 47-76.

[24] S. Ghodrat, M. Janssen, R. H. Petrov, L. A. I. Kestens, J. Sietsma, Microstructural Evolution of Compacted Graphite Iron under Thermo-Mechanical Fatigue Conditions, Advanced Materials Research 409 (2012) 757-762.

[25] S. G. S. Raman, D. Argence, A. Pineau, High temperature short fatigue crack behaviour in a stainless steel, Fatigue \& Fracture of Engineering Materials \& Structures 20 (7) (1997) 1015-1031.

[26] B. Fournier, M. Sauzay, C. Caës, M. Noblecourt, M. Mottot, A. Bougault, V. Rabeau, A. Pineau, Creep-fatigue-oxidation interactions in a $9 \mathrm{Cr}-1 \mathrm{Mo}$ martensitic steel. Part I: Effect of tensile holding period on fatigue lifetime, International Journal of Fatigue 30 (4) (2008) 649-662.

[27] B. Fournier, M. Sauzay, C. Caës, M. Noblecourt, M. Mottot, A. Bougault, V. Rabeau, A. Pineau, Creep-fatigue-oxidation interactions in a $9 \mathrm{Cr}-1 \mathrm{Mo}$ martensitic steel. Part II: Effect of compressive holding period on fatigue lifetime, International Journal of Fatigue 30 (4) (2008) 649-662.

[28] R. Neu, H. Sehitoglu, Thermomechanical fatigue, oxidation, and creep: Part II. Life prediction, Metallurgical Transaction A 20 (9) (1989) 1769-1783.

[29] R. Prescott, M. J. Graham, The Oxidation of Iron-Aluminum Alloys, Oxidation of Metals 38 (1992) 73-87. 